\title{
El nuevo humanismo en la novela contemporánea francesa
}

\author{
Monique LANDAIS CHOIMET \\ Universidad Nacional Autónoma de México
}

Si se plantea la razón de ser del hombre en función de su apertura al otro, cabe privilegiar la lectura de una novelista francesa contemporánea llamada Sylvie Germain, quien destaca por el enfoque ético levinasiano que le da a su obra.

Esta perspectiva insólita para nuestra época recalca la firme voluntad de ir a contracorriente. De hecho, en sus personajes figuran las presentes plagas: abandonados, marginados y pordioseros, y dejan entrever la indiferencia colectiva frente a su indigencia. Pero, lejos de caer en el deprimismo, la novelista reencanta con admirable maestría poética la esperanza nutrida por la responsabilidad ajena.

Ponerse a la escucha atenta del prójimo es reparar la injusticia originaria en un acto de militancia necesaria para la reconstrucción de la comunidad humana.

PAlabras Clave: novela contemporánea, nuevo humanismo, Sylvie Germain, Emmanuel Levinas.

Regarding man's raison d'être in relation to his openness towards the other it is worth reading French contemporary novelist Sylvie Germain known for the Levinasianan-ethical perspective of her work.

This perspective, unusual in our time, reinforces the steady determination of going against the grain. As a matter of fact, her characters personify current calamities: the forsaken, outsiders, beggars; they speak of the collective indifference aroused by their poverty. However far from yielding to deprimism, the novelist depicts with outstanding poetical skill the hope that is nourished by mutual responsibility.

To be in the disposition of patiently listening to our brethren is a way of making up for this primal injustice. It is a necessary act of militancy for the reconstruction of the human community.

KEY WoRDS: Contemporary novel, New Humanism, Sylvie Germain, Emmanuel Levinas.

La verdad de la metafísica se produce en las obras.

Emmanuel Levinas

Al igual que un casco de meteorito, con el cual lo compara la autora, Magnus irrumpe en nuestras vidas de manera abrupta y misteriosa. En efecto, ignoramos la proveniencia 
del protagonista pero sabemos que tiene un origen que le es propio, específico y que lo hace, de alguna manera, único en cuanto a categoría se refiere. Las comparaciones ulteriores nos confirman nuestra intuición cuando leemos a continuación que una esquirla nos revelará algún dato concerniente a la fauna y que un jirón de papiro o un trozo de cerámica nos guiarán hacia una civilización perdida. Fragmentos todos de existencias que se inscriben en el infinito del mundo. ¿Acaso debemos entonces percibir a Magnus como si fuera un ser en peligro de extinción, de quien se encontraron por suerte algunos signos durante una de estas excavaciones practicadas por científicos persistentes convencidos de la utilidad de su labor y empeñados en sacar a relucir arcanos que estiman de vital importancia para nuestro futuro y sobrevivencia? ¿O será que necesitamos investigar huellas hoy olvidadas pero que vale la pena traer a colación para recordar datos valiosos pero despreciados por nuestra memoria selectiva al servicio de una época pragmática que descarta cualquier dato fútil, inútil, en el sentido que no sirve a ningún fin político, económico o mercantil? En este horizonte, más allá del humanismo clásico o de la desesperanza existencial, comienza a perfilarse un nuevo humanismo: el humanismo del otro hombre. "Humanismo que se preocupa más del hambre y de la miseria de los otros que de resguardar la propiedad, la libertad y la dignidad de la misma subjetividad" (Levinas, 2009: 5).

Como bien lo entendemos, se nos presentan nuevos derroteros, los cuales partirán del hombre para llevamos al hombre en su plenitud, es decir, sin atribuirle características que no le son propias pero que le fueron impuestas a lo largo de los siglos por necesidades creadas y que hoy, de alguna manera, opacan o hasta ciegan nuestras relaciones humanas. Accesorios tales como el dinero, fuente de supuesto éxito y poder; la apariencia física y sus adornos tales como cosméticos, vestimenta, joyas, ostentosidad sustituta de autenticidad; el coche o la casa, marcas de estatus social y de supuesta respetabilidad. Es menester subrayar, en este preciso momento de nuestra reflexión, la firme voluntad por parte de Sylvie Germain de despojar a sus personajes, sean éstos principales o secundarios, de cualquiera de estos artificios. Queda clara entonces la imperante necesidad de entender el punto de vista adoptado por el filósofo y su discípula.

Por un lado, el hombre considerado aquí es el hombre en sí, fuera de cualquier marco espacio-temporal fijo que podría limitarlo a un periodo o un lugar específico de la Historia. Así, nos llevan nuestros pensadores al meollo de la problemática: cualquier tema ahí tratado concierne al hombre en tanto creatura, o sea el ser infinito sin origen ni final, único en toda su misteriosa complejidad. El siguiente poema de Jules Supervielle le permitió ilustrar a Sylvie Germain, de manera ingenua pero persuasiva, esta desnudez humana, alegoría primera del Otro, encamada en la mitología bíblica bajo el nombre de Adán y Eva:

Un boeuf gris de la Chine Couché dans son étable, Allonge son échine Et dans le même instant Un boeuf de l'Uruguay
Vole sur l'un et Vautre

A travers jour et nuit

L'oiseau qui fait sans bruit

Le tour de la planète

Et jamais ne la touche 
Si quelqu'un a bougé.

Nos percatamos que la referencia al bestiario, muy frecuente en las novelas de esta autora, remite al género didáctico de la fábula, muy preciado por los moralistas como Esopo o La Fontaine por su sencillez, su claridad y su inocencia en cuanto a la temática tratada. Este poema concuerda con la intención de llevar la reflexión a un nivel superior, más allá de la condición terrenal atiborrada de contingencias abrumadoras. El pájaro viajero simboliza acaso la altura a la que tenemos que acceder para sustraemos a los numerosos y repentinos peligros que nos acechan.

Por otro lado, la simbología ve también en el pájaro, ser celeste, una suerte de embajador entre el más allá y el mundo terrenal, distinguido aquí como una especie de huella intangible pero perceptible al oído fino del ser primitivo entendido como puro, libre de cualquier influencia engañosa, curioso y ávido de conocimiento, en busca de la verdad conmovedora por la que sí, definitivamente, vale la pena vivir. Estamos hablando del duro nudo del "único" en mí, de la fuente del amor para el Otro que el filósofo judío de origen lituano, sobreviviente de los campos de concentración nazis, se empeñó en definir y enseñar a los jóvenes universitarios durante el resto de su vida con el fin de militar por la fuerza de la Verdad y la esperanza de un entendimiento entre los hombres (Levinas, 2009: 42).

En este punto, podemos formular la significación muy peculiar que el filósofo le da a la presencia-ausencia de la huella. Semejante al pájaro invisible, es incorpórea y etérea, obícua y omnisciente, eterna e infinita. En este caso, ¿dónde y cómo podemos encontrarla y, más todavía, interpretarla? En el Otro precisamente presente en cada uno de nosotros, en nuestro interés por el otro, por acercamos a él en esta proximidad de la piedad y de la generosidad: "La huella como huella no conduce solamente hacia el pasado, sino que es el paso mismo hacia un pasado más lejano que todo pasado y que todo porvenir, los cuales se ordenan aún en mi tiempo - hacia el pasado del Otro donde se perfila la eternidad-, pasado absoluto que reúne todos los tiempos. [...] Pero en la huella del Otro resplandece el rostro" (Levinas, 2009: 75).

De antemano, la creación de la novela que aquí nos ocupa cumple ese mismo papel revelador de verdades o semiverdades acerca de la Historia del universo y de la humanidad. Así, nos situamos claramente en un continuum infinito y abierto de creación, por una parte, y de investigación, por otra parte, del que, si bien ignoramos el origen, no dudamos ni un solo instante que proviene de épocas suficientemente remotas para que la memoria falible del hombre no las recuerde. Así, el trabajo de un escritor comprometido, como lo concibe Sylvie Germain, consiste precisamente en crear y decriptar estas huellas dejadas por los hombres a lo largo de sus vidas. Su trabajo tendrá entonces como fin traer a nuestro presente los conocimientos faltantes indispensables que puedan surgir del pasado para permitir la inteligibilidad de la especie que sólo nos será accesible en la medida que será representada por medio de una anécdota. En efecto, es el ser en acto, su acontecimiento, su experiencia en interacción con los demás, que nos permitirán 
comprender, realizar y pregonar esta filosofía tan valiosa en nuestra época propicia para cambios profundos. En esta doble labor reside la tarea del filósofo de la ética tanto como de la novelista humanista que viene expuesta de la siguiente manera:

Une rumeur montée des confins du passé pour se mêler à celle affluant de toutes parts du présent. Un vent de voix, une polyphonie de souffles. En chacun la voix d'un souffleur murmure en sourdine, incognito - voix apocryphe qui peut apporter des nouvelles insoupçonnées du monde, des autres et de soi-même pour peu qu'on tende l'oreille. Écrire, c'est descendre dans la fosse du souffleur pour apprendre à écouter la langue respirer là où elle se tait, entre les mots, autour des mots, parfois au cœur des mots (2005: 12).

Al emprender la lectura de esta novela, nos comprometemos entonces, a la par de su autora, a abrimos al otro, al ser humano que somos nosotros mismos, de manera muy atenta como nos lo aconseja de modo insistente al intitular el íncipit metodológico, Ouverture. Apertura en el tiempo, apertura en el espacio, apertura hacia lo ajeno como principio primero, sin prejuicio ni egocentrismo, como actitud generosa para recibir a la obra que se nos presenta. Este orden infinito exige una cuidadosa explicación léxica llevada a cabo a guisa de glosario por el mismo filósofo. Distingue tres niveles de aprehensión del hombre inconfundibles, estrechamente ligados los unos a los otros por una relación de complementariedad.

Procede a la distinción del nivel primero del ente, Seiendes en alemán, que corresponde al ens de los escolásticos, al que llamamos más arriba el acontecimiento del ser entendido como la gravidez del ser. Luego, de este nivel propiamente terrenal, nos elevamos al nivel ontológico verbalizado como esencia, correspondiente al Sein alemán y al esse latino y equivalente a la manera de relacionarse de las entidades existentes. Finalmente, introduce Levinas un tercer nivel en el que pone precisamente en tela de juicio la razón que rige estas interrelaciones (Levinas, 2001: 13) y, a falta de atreverse a crear el neologismo essance, a la manera de Jacques Derrida, quien creó el término différance, se limita a precisar que en sus escritos esencia se entenderá como eidos, es decir, la contemplación pura o quididad (Levinas, 2003: 41).

\section{El sujeto definido a partir del finito del ser}

La manifestación del Mal perpetrado durante el Holocausto con una crueldad llevada a extremos nunca antes imaginados hizo que el filósofo tomara conciencia de la naturaleza muy preocupante de nuestra época. Consideró que vivíamos una situación de emergencia tal que urgía recordar ciertos principios éticos antes de que fuera, de nuevo, demasiado tarde. Había que reconsiderar la relación del ser humano con la Razón, con el Bien y el Mal, con el conocimiento. Apremiaba colmar el nuevo vacío intelectual y moral que imperaba en nuestro mundo después de la declaración por la filosofía occidental nietzscheana de la muerte de Dios. Al igual que el Fausto decepcionado 
por los múltiples fracasos del hombre moderno, el ser contemporáneo se entregó al diablo en su furia de destrucción y su obsesión por la muerte, cegado por las tendencias maléficas tales como el egoísmo, el hedonismo y la individualidad, antivalores que bastan para acreditar el instinto de sobrevivencia propia cuando cualquier medio justifica el fin pensado.

Por consiguiente, era necesario incitar al ser a reflexionar para que fuera más humano, característica que lo distingue precisamente del lobo hobbesiano. Empero, importaba ante todo no recaer en las mistificaciones religiosas consideradas una falacia y una imperdonable hipocresía de las que nadie podía dudar al recordar que la Iglesia católica había apoyado y luego ignorado el régimen hitleriano. Los espíritus se encontraban demasiado dolidos como para volver a adoptar la lengua, las plegarias o los rituales de dicha institución. Así es como Emmanuel Levinas optó por un lenguaje laico de índole ético a fin de emprender una reflexión militante concerniente a las relaciones humanas hoy desnaturalizadas.

Caminando en los pasos de su maestro, Sylvie Germain les dio vida a unos personajes cuyo papel consistía en ejemplificar estos antivalores antes mencionados para que el lector sintiera en su propio ser, gracias al proceso catártico, el horror y la piedad, sentimientos que nos son devueltos por las verdaderas tragedias clásicas que son sus novelas. A la diferencia capital que el lector que somos tiende, tras la lectura, a aproximarse a este ser fundamentalmente desdichado que es el personaje germaniano, para desarrollar cierta sensibilidad en el vivir cotidiano.

El infinito experimentado en el finito del ser nos permite, cual procedimiento pedagógico, entender mejor nuestra condición y nos revela la importancia vital de la literatura en cuanto a adquisición de conocimientos $\mathrm{y}$, de ser posible, prevención de nuevos holocaustos, verdaderos suicidios colectivos. Veamos entonces, con detenimiento, cómo la autora procede para revelamos la problemática humanista que se plantea al hombre contemporáneo. Nos dedicaremos al estudio de tres aspectos fundamentales: la estructura de la novela, la noción de personaje y la lengua poética.

\section{La novela palimpsesto}

La novela intitulada Magnus que escribe Sylvie Germain en 2005 y que fuera galardonada con el premio Goncourt des Lycéens responde a diversas inquietudes dentro de las cuales la primera sería lo que la propia autora llama el deber de memoria. Esta línea de investigación literaria cobra distintos matices al empezar la obra con un primer encabezado a guisa de título para el primer capítulo, Ouverture. Sabemos que una apertura se connota, antes que nada, como reciprocidad por medio de una invitación a compartir una experiencia, una aventura, un viaje tal vez iniciático. De hecho, Levinas le atribuye tres sentidos a este término polisémico: 
designar también la intencionalidad de la conciencia-un éxtasis en el ser. Éxtasis de la ek-sistencia, según Heidegger, que anima la conciencia, que está llamada, por la apertura original de la esencia (del Sein), a ocupar un lugar en ese drama de la apertura. [... ] Pero la apertura puede tener un tercer sentido. No es ya la esencia del ser que se abre para mostrarse, ni la conciencia que se abre a la presencia de la esencia abierta y confiada en ella. La apertura es lo descamado de la piel expuesta a la herida y al ultraje. La apertura es la vulnerabilidad de una piel ofrecida, en el ultraje y en la herida, más allá de todo lo que puede mostrarse, más allá de todo lo que, de la esencia del ser, puede exponerse a la comprensión y a la celebración (Levinas, 2009: 122).

Es del todo fácil ahora adentramos en la novela, en la historia narrada, sabiendo que el tema ahí tratado nos atañe directamente y que la escritora, siguiendo las huellas del filósofo, la escribió en beneficio nuestro como si fuera una obra humanitaria. Seguramente es también una bienvenida en forma de guiño simpático para invitar al joven lector a adentrarse en el drama sin ningún temor. Al desarrollarse la obra, por las múltiples referencias intertextuales de todo género cual prisma que refracta los diversos colores, se abre el texto narrativo hacia otras dimensiones que lo amplifican de manera infinita. Su estructura es la de un hipertexto, diseñada a imagen y semejanza de la red internet y de su usuario, el hombre contemporáneo, quien se enriquece inmensamente al abrir las puertas multidisciplinarias que se le presentan. Así es como la autora organiza su relato de tal manera que sea el punto de partida hacia fuerzas centrífugas que trazan senderos cual pistas de búsqueda, con el fin de otorgarle al tema toda su magnificencia, su magnitud. Ahí reside la primera razón de ser del título Magnus, que nos dice claramente la supremacía del hombre sobre la tierra, centro de interés esencial de los Humanistas a través de los siglos.

Es menester precisar que Sylvie Germain, quien se formó con Emmanuel Levinas, es doctora en filosofía por la universidad de La Sorbona. Entonces, Magnus funge como una obra de divulgación, de vulgarización de la filosofía de la ética dirigida hacia un público neófito en la materia. Se perfila ahí el primer palimpsesto que resulta ser el fundamental puesto que la autora reescribió, por así decirlo, la filosofía de la ética levinasiana, mas bajo forma de una extensa parábola. Pareciera que, frente al rechazo demasiado frecuente de la lectura seria por parte de los jóvenes, al déficit de las carreras humanistas en las universidades, al terror mediático esclavizante de espíritus y almas, Sylvie Germain decidió poner su pluma al servicio de la causa literaria. Decididamente, se acercó a los jóvenes y los invitó a abrir su libro; lo que hicieron y disfrutaron, sin ninguna duda, ya que la premiaron. De alguna manera, rindieron un homenaje a Levinas, quien falleció el mismo año de la entrega del premio. En esta vida que le otorgaban a la obra por medio de su lectura, cual fuego prometeico, le concedieron un nuevo impulso a la filosofía de la ética, por lo menos en sus dos principios sociales, la proximidad y la responsabilidad. En virtud de la influencia que pueda tener una novela sobre su lector y a falta de grandes cambios repentinos, podemos siquiera esperar que esta primera apertura literaria sea el primer paso hacia un habitus de lectura y de reflexión. 
Conviene agregar a esta actitud proselitista germaniana un segundo procedimiento que le permite instalar una serie de anzuelos para enganchar al lector acostumbrado al hipertexto. En efecto, al definir la historia que nos va a relatar como si fuera un cuento, Sylvie Germain circunscribe nítidamente la parte ficticia, divertida, en el sentido pascaliano, de su novela, de tal suerte que podemos proceder a la lectura de la trama entretenida, en sí la aventura, basándonos únicamente en los llamados Fragmentos. Correspondientes a cierta tendencia literaria actual que describe o representa solamente fracciones de vida, este recurso ilustra cierta postura realista desde varios puntos de vista. Se habrá notado que "les mots d'un livre ne forment pas davantage un bloc que les jours d'une vie humaine, aussi abondants soient ces mots et ces jours, ils dessinent juste un archipel de phrases, de suggestions, de possibilités inépuisées sur un vaste fond de silence. [...] Un vent de voix, une polyphonie de soufflés" (2005: 12).

¿Qué mejor que esta apertura a la tolerancia para seducir a un joven, a un adolescente en guerra contra cualquier imposición o dogma? Estos fragmentos breves coinciden también con su capacidad de atención, no le robarán mucho tiempo. Siguiendo esta guía narrativa, descubrirá al filo de una lectura amena y descansada, las trágicas aventuras de un protagonista, que, a pesar de muchos tropiezos, logra sobrevivir y seguir adelante. La intriga asegura un final feliz catártico; al final de cuentas, nos queda la vida y esto es lo más preciado. Empero, estos fragmentos se asemejan, por otra parte, a los versículos bíblicos, parábolas breves con fin didáctico en la medida que exigen reflexión e interpretación. Y si esto está correcto, se plantea entonces la tarea de emprender otro camino más complejo y arduo pero, a su vez, mucho más enriquecedor. Podríamos pensar, de hecho, en los exploradores que descubren paisajes inesperados y maravillosos al querer llevar una búsqueda fuera de los caminos trillados. Y es precisamente al descubrimiento de extraordinarios senderos a donde nos quiere llevar Sylvie Germain, itinerarios despreciados hoy en día por una sociedad materialista, ávida de placeres inmediatos que satisfacen el espejismo del instante, la ilusión adormecedora que complace al individualismo indiferente e insensible de nuestra época. Disipados, presas de mil y una distracciones enajenantes, nos hemos alejado del silencio necesario para volver a oír voces que claman su existencia bajo el clamor mediático; voces calladas, mas no muertas, que revelan su origen a quien se detiene a escuchar como Magnus. "Rien de plus - aucun flamboiement, aucune agitation du corps assoupi, aucun râle ni bredouillement proférés par sa bouche. Juste ce souffle montant avec lenteur, avec ampleur, des profondeurs du corps concentré à l'extrême non sur lui-même, mais sur l'oubli de soi- sur une excavation, un évidement de soi" (2005: 266).

Las múltiples referencias bíblicas enjambradas a lo largo de la novela, al igual que la inserción de numerosas citas multigenéricas, son otros tantos libros abiertos que reclaman la atención para sí mismos, reivindicando un lugar equiparable o hasta superior a la novela que estamos leyendo, como lo recalca la alusión frecuente al livre des merveilles et de l'insoupçonné (2005: 275). Mientras Magnus emprende un largo viaje en busca de su identidad perdida, el lector se lanza por su parte a la persecución del significado de todas estas bifurcaciones propuestas antes y después de cada fragmento. 
Aquellas digresiones arman, de facto, una especie de red significante alrededor del relato y no cabe duda que la autora nos lleva de la mano a través de un laberinto que se parece al andar humano en la vida, es decir, un errar en la tierra con todos los riesgos que el solo caminar conlleva, al igual que la sed insaciable de conocimientos.

Paralelamente al desarrollo del argumento aparecen en primer lugar y de manera puntual las notitas o apostillas que aportan elementos referenciales como genealogías verdaderas o ficticias pero siempre verosímiles, o bien datos históricos reales, que enmarcan la ficción dentro del contexto histórico de la Segunda Guerra Mundial, procedimiento cuyo efecto realista sumerge al lector en una época conflictiva no muy remota pero, sí, olvidada por la mayoría. En segundo lugar, se suman al relato las secuencias que agregan al texto primario citas poéticas, teatrales, musicales, cinematográficas, o concernientes a la simbología, todas ellas floreciendo el imaginario artístico. En tercer lugar y más en la segunda mitad de la obra, se apresuran las efemérides como homenaje a los mártires, las resonancias a través de los tiempos para recordar la lucha eterna del hombre en contra de la opresión y la injusticia en pos de una vida digna, los ecos que unen los países más allá de las distancias en una misma empresa de edificación del ser, una letanía cual oración recitada implorando piedad y compasión frente al silencio divino. Finalmente, se impone un palimpsesto refiriendo palabras en boca de tres rabinos que reflexionan sobre de otro modo que ser o más allá de la esencia, podríamos decirlo así para retomar el título de un ensayo de Emmanuel Levinas. Originalidad literaria tanto a nivel de la estructura como de la temática y de la simbología, Magnus abre un diálogo plural a través del tiempo y del espacio a manera de un infinito palimpsesto.

La totalidad del ser en la que el ser resplandece como significación, no es una entidad fijada eternamente, sino que requiere el ordenamiento y la reunión, el acto cultural del hombre. El ser en su conjunto - la significación - brilla en las obras de los poetas y de los artistas. Pero brilla de diversas maneras en los diversos artistas de la misma cultura y se expresa diversamente en las culturas diversas. Esta diversidad de expresión no traiciona, para Merleau-Ponty, al ser, sino que hace centellear la inagotable riqueza de su acontecimiento (Levinas, 2009: 134).

Esta suerte de descriptivo estructural revela los tres niveles de elaboración literaria recurrentes en las novelas de Sylvie Germain, principios teóricos que buscan reunir diversas disciplinas humanísticas en un afán de recrear la novela francesa contemporánea. De hecho, todo texto resulta ser un palimpsesto, en este sentido, ya que, para ser reescrito a lo largo de los siglos, la historia de la Humanidad necesita ser borrada repetidamente y vuelta a escribir, sin que nunca se llegue ni al verdadero origen. Quien se refiere a la ausencia del principio, sobreentiende a su vez la falta absoluta del final, instituyendo así la naturaleza abierta del palimpsesto. ${ }^{1}$ Esta inconclusividad constitu- 
ye precisamente la esperanza, el horizonte abierto hacia el infinito que nos brinda la escritora. A imagen y semejanza de Ezequiel hablando de Job, nos toca avaler nuestro propio libro para empezar a escribirlo, alegoría de nuestra propia vida vivida en plena conciencia. Palimpsesto pasado y futuro, alegoría de nuestro ser en devenir.

Consideraremos al protagonista-sujeto en cuanto experiencia de vida individual y colectiva, inscrita en un marco sociohistórico muy particular; lo contemplaremos de igual manera como acontecimiento del ser por medio de su propia trascendencia para, finalmente, apreciar lo que Levinas llama el Deseo del Otro en el olvido de sí mismo: "El deseo de Otro - la socialidad - nace en un ser al que no le falta nada o, más exactamente, nace más allá de todo lo que puede faltarle o satisfacerle. En el Deseo, el Yo se dirige hacia el Otro, comprometiendo la soberana identificación del Yo consigo mismo... La relación con el Otro me cuestiona, me vacía de mí mismo y no cesa de vaciarme al descubrirme recursos siempre nuevos" (Levinas, 2009: 56).

En nuestra sociedad imagenóloga obsesionada por la apariencia, algunos pensadores se esmeran en recordamos los valores propios del Nuevo Humanismo, enfocados hacia la ética. El maestro y su discípula nos ilustran, cada quien a su manera, para proporcionamos el conocimiento, clave de nuevos horizontes. Emmanuel Levinas no está al alcance de cualquier lector aun cuando ciertos textos suyos, como Humanismo del otro hombre, parecen tomar en cuenta al lector novato, mientras que Sylvie Germain, a su vez, nos regala con Magnus una verdadera novela de iniciación a la filosofía. Se habrá notado así, por ambas partes, la estimable voluntad de cultivar, por así decirlo, al lector no iniciado.

El esfuerzo patente que certifica la calidad de una obra tan prolifica me parece merecer, de hecho, un idéntico ardor al estudiarla. Es de admirar la maravilla poética que surge de las páginas del filósofo como de la novelista. Sin un profundo amor a las Letras, las palabras, el Verbo, no hubieran podido lograr semejante estética. Seguiremos siendo conscientes del grado de exigencia que implica la confrontación de esas dos grandes figuras contemporáneas, eméritas.

\section{Obras citadas}

Germain, Sylvie. 2005. Magnus. París: Albin Michel.

Goulet, Alain. 2008. L'univers de Sylvie Germain. Caen: Presses Universitaires de Caen.

LEVINAS, Emmanuel. 2009. Humanismo del otro hombre. México: Siglo XXI. . 2003. De otro modo que ser. Salamanca: Ediciones Sígueme. 2001. Entre nosotros. Valencia: Pre-textos. 